\title{
Влияние топологии молекул на сорбцию производных 1,2,4-триазола и 1,2,4-триазина неполярными сорбентами в условиях жидкостной хроматографии
}

\author{
Карасева И.Н., Карасев М.О., Курбатова С.В. \\ Самарский национальный исследовательский университет им. академика С.П. Королева, Самара
}

Поступила в редакцию 17.09.2018 г.

DOI: https://doi.org/10.17308/sorpchrom.2018.18/618

Исследована зависимость хроматографического удерживания производных 1,2,4-триазина и 1,2,4-триазола из водно-ацетонитрильных растворов на октадецилсиликагеле, пористом графитированном углероде и сверхсшитом полистироле от строения молекул сорбатов. Получены корреляции между топологическими индексами, физико-химическими характеристиками и факторами удерживания указанных соединений для условий обращенно-фазовой высокоэффективной жидкостной хроматографии.

Ключевые слова: обращенно-фазовая высокоэффективная жидкостная хроматография, производные 1,2,4-триазина и 1,2,4-триазола, октадецилсиликагель, сверхсшитый полистирол, пористый графитированный углерод, топология, индексы Рандича, индексы Винера.

\section{Influence of the topology of molecules on the sorption of derivatives of 1,2,4-triazole and 1,2,4-triazine by nonpolar sorbents under conditions of liquid chromatography}

\author{
Karaseva I.N., Karasev M.O., Kurbatova S.V. \\ Samara National Research University named after Academician S.P. Korolev, Samara
}

The article is devoted to investigation of 1,2,4-triazine and 1,2,4-triazole derivatives chromatographic behavior. The purpose of this work was to study the regularities of some 1,2,4- triazine and 1,2,4triazole derivatives sorption from water - acetonitrile solutions on different nature sorbents under condition of reversed-phase high performance liquid chromatography. Octadecyl silica gel (ODS), porous graphitized carbon (PGC) and hypercrosslinked polystyrene (HCLP) were used as sorbents. As a result, correlations between topological indices, physico-chemical characteristics, and retention factors of these compounds were obtained. As it was shown, the main factors influencing the properties of the triazole and triazine derivatives studied by us are the presence and number of heteroatoms, the nature of the substituents and their position, which to a large extent also determine the topology of the molecules. The analysis of the correlations showed that, in general, connectivity indices (ICs) of all five orders correlate well with the 1,2,4-triazole and 1,2,4triazine, 1,3-benzoxazole derivatives with parameters characterizing the dispersion interactions. The maximum values of the correlation coefficient are characteristic for zero-order correlations of the linkage index, the minimum values for the third order $3 \chi$. The values of the Wiener index (IV) in the series of heterocycles investigated increase monotonically with the increase in the size of the molecules; however, the correlation between the IV and the dimensional parameters of the molecules turns out to be somewhat less rigorous than for the IC. It was found that the replacement of two methyl radicals by two phenyl derivatives of 1,2,4triazines significantly increases the surface area, molecule volume and topological indices. However, the 
retention values vary depending on the nature of the sorbent. The likely reason for the apparent discrepancy between the values of the retention factor and the dimensional characteristics of some substances may be the stereochemistry of the molecules, which is not taken into account both in the physicochemical and topological parameters used.

In conclusion, the accumulation of experimental data on the retention of sorbates of this type will solve the problem of establishing the relationship between sorption characteristics and other physicochemical parameters and molecular structure.

Keywords: RP HPLC, 1,2,4-triazine and 1.2.4-triazole derivatives, octadecyl silica, hypercrosslinked polystyrene, porous graphitized carbon, topology, connectivity indices(Randic), Viner indices.

\section{Введение}

Разнообразие межмолекулярных взаимодействий (ММВ), проявляющихся в хроматографической системе в условиях жидкостной хроматографии и обусловленных присутствием активных с точки зрения таких взаимодействий сорбента и элюента, создает предпосылки для установления взаимосвязи между строением молекул аналитов, участвующих в этих МMB, и их удерживанием на поверхности сорбента. Алгоритм решения обратной задачи - по сорбционным характеристикам оценить особенности строения сорбируемых молекул - хорошо представлен в трудах А.В. Киселева при описании его хроматоскопического метода для газовой хроматографии [1]. В условиях жидкостной хроматографии на удерживание влияет множество таких факторов, как природа сорбента, качественный и количественный состав элюента, его $\mathrm{pH}$, возможность протекания процессов ассоциации молекул сорбатов и компонентов подвижной фазы или их ионизации в объемном растворе элюента и т.п. С одной стороны, все эти факторы существенно осложняют установление взаимосвязи между строением молекул сорбатов и их удерживанием, а с другой - предоставляют для этого еще более широкие возможности, обусловленные широким спектром межмолекулярных взаимодействий, реализуемых за счет варьирования природы самой хроматографической системы и условий хроматографирования в целом [2]. Фундаментом решения подобных задач является накопление массива данных по удерживанию веществ различного строения в разнообразных условиях хроматографирования.

В предыдущих публикациях мы сделали некоторые шаги в направлении установления взаимосвязи между строением азотистых гетероциклов и их удерживанием в условиях высокоэффективной жидкостной хроматографии (ВЭЖХ) на примере производных бензотриазола и 1,2,3,4-тетрагидрохинолина [3, 4]. Особенности строения азотистых гетероциклов связаны, прежде всего, с их полифункциональностью, наличием одного или нескольких гетероатомов, электронодефицитных или электроноизбыточных ароматических фрагментов, с вариациями стереохимических и топологических характеристик молекул, приводящих к появлению соответствующих особенностей хроматографического удерживания этих соединений [5]. Учет таких особенностей при исследовании взаимосвязи между строением и хроматографическим удерживанием азотистых гетероциклов в условиях ВЭЖХ в конечном итоге и позволяет решать двуединую задачу - с одной стороны, прогнозировать удерживание этих веществ на основе данных об их строении и физико-химических характеристиках, и, с другой, по величинам удерживания делать предположения о структуре соединений. Анализ особенностей хроматографического удерживания азотистых гетероциклов, представленный нами в публикациях $[3,4]$ показал, что при сорбции этих соединений в условиях обращенно-фазового варианта жидкостной хроматографии, особенно на сорбентах с плоской поверхностью, весьма важную роль играет топология молекулы. При этом существование корреляций между сорбционными и топологическими характеристиками позволяет прогнозировать величины удерживания с весьма приемлемой точностью.

Карасева и др. / Сорбционные и хроматографические процессы. 2018. Т. 18. № 6 
Яркими представителями класса азотсодержащих ароматических гетероциклов являются триазолы и триазины, обладающие широким спектром важных с практической точки зрения свойств. Прежде всего, следует отметить их высокую биологическую активность, включая противомикробную, фунгицидную, противоопухолевую, противовоспалительную и многие другие. Производные этих гетероциклов находят применение в медицине, сельском хозяйстве, разнообразных технологических и биологических процессах [6-8]. Еще один аспект их практического использования связан с развитием химии координационных полимеров, в структуру которых в качестве лигандов входят производные триазолов. С начала нового тысячелетия отмечен экспоненциальный рост публикаций по тематике, связанной с химией координационных полимеров на основе азолов и азотистых гетероциклов в целом [9-12]. Неудивительной поэтому является интенсификация развития различных методов исследования подобных соединений, среди которых важное место отводится жидкостной хроматографии. Применение этого метода позволяет не только анализировать различные вещества и их смеси вне зависимости от агрегатного состояния и летучести, но и осуществлять одновременно разделение и идентификацию соединений, что оказывается особенно актуальным при анализе биологически активных веществ и лекарственных препаратов на их основе.

В связи с изложенным выше, целью настоящей работы явилось исследование взаимосвязи физико-химических и топологических параметров производных 1,2,4триазола и 1,2,4-триазина и их хроматографического удерживания в условиях обращенно-фазовой высокоэффективной жидкостной хроматографии (ОФ ВЭЖХ).

\section{Эксперимент}

Хроматографический эксперимент проводили на жидкостном хроматографе «Varian ProStar» с УФ-спектрофотометрическим детектором ProStar 325 и плунжерным насосом - ProStrar 210 при длине волны 254 нм. В качестве адсорбентов использовали октадецилированный силикагель «Waters» (размеры колонки 3.9×150 мм, размер частиц сорбента 5 мкм), коммерческий сверхсшитый полистирол марки MMN1 (размеры колонки 150x4.6 мм, размер зерен сорбента 5 мкм) и пористый графитированный углерод - гиперкарб (размеры колонки 50х3 мм, размер зерен сорбента 5 мкм). Элюирование осуществляли в изократическом режиме при комнатной температуре. В качестве подвижной фазы применяли смеси ацетонитрил - вода с концентрацией ацетонитрила от 40 до $80 \%$ (об). Объемная скорость потока элюента составляла 500 мкл/мин. Растворы сорбатов готовили растворением сухих индивидуальных веществ в подходящих растворителях. Объем вводимой пробы составлял 20 мкл. В качестве несорбирующегося вещества использовали нитрит натрия.

Удерживание веществ характеризовали величиной фактора удерживания $(k)$, рассчитанной по известным формулам [13].

С использованием программы НyperChem версии 8.0.8 полуэмпирическим методом РМ3 с полной оптимизацией геометрии молекул были рассчитаны мольный объем $(V)$, дипольный момент $(\mu)$, поляризуемость $(\alpha)$, липофильность $(\lg \mathrm{P})$, молярная рефракция (MR), площадь поверхности молекул $\left(\mathrm{S}_{\mathrm{Grid}}\right)$ производных $1,2,4-$ триазола и 1,2,4-триазина, значения которых приведены в таблице 1.

Расчет индексов связанности (ИС) нулевого - пятого порядков производных 1,2,4-триазола и 1,2,4-триазина осуществляли по формулам:

индекс связанности нулевого порядка ${ }^{0} \chi=\sum\left(\delta_{\mathrm{i}}\right)^{-1 / 2}$,

индекс связанности первого порядка ${ }^{1} \chi=\sum_{q=1}^{K}\left(\left(\delta_{\mathrm{i}} \delta_{\mathrm{j}}\right)^{-1 / 2}\right.$,

Карасева и др. / Сорбционные и хроматографические процессы. 2018. Т. 18. № 6 
где $\delta_{\mathrm{i}}, \delta_{\mathrm{j}}-$ значения $\delta$ для двух атомов связи $q ; i, j-$ атомы, формально связанные в молекуле рассматриваемой связью; К- общее число связей в молекуле.

Таблица 1. Некоторые физико-химические характеристики исследованных соединений

\begin{tabular}{|c|c|c|c|c|c|c|}
\hline № & Формула & $S_{\text {grid }} \AA^{\circ}$ & $V, \AA^{3}$ & $\lg P$ & $\alpha, \AA^{3}$ & $\mu, D$ \\
\hline 1 & 2 & 3 & 4 & 5 & 6 & 7 \\
\hline 1 & & 407.44 & 633.77 & 0.85 & 22.92 & 6.41 \\
\hline 2 & & 472.49 & 769.90 & 2.20 & 30.19 & 5.32 \\
\hline 3 & & 566.80 & 964.30 & 4.69 & 40.56 & 6.08 \\
\hline 4 & & 309.41 & 455.50 & 1.17 & 15.26 & 3.78 \\
\hline 5 & & 333.36 & 503.20 & 1.45 & 17.09 & 4.34 \\
\hline 6 & & 387.96 & 604.40 & 1.82 & 20.76 & 5.32 \\
\hline 7 & & 524.76 & 877.0 & 4.41 & 34.58 & 5.07 \\
\hline 8 & & 300.95 & 440.50 & 0.22 & 14.55 & 3.37 \\
\hline 9 & & 513.70 & 867.30 & 3.46 & 33.87 & 4.94 \\
\hline 10 & & 422.44 & 678.0 & 2.24 & 26.20 & 3.72 \\
\hline 11 & & 575.50 & 990.90 & 5.11 & 41.85 & 3.92 \\
\hline
\end{tabular}




\begin{tabular}{|c|c|c|c|c|c|c|}
\hline 1 & 2 & 3 & 4 & 5 & 6 & 7 \\
\hline 12 & & 392.94 & 625.8 & 2.15 & 24.36 & 3.69 \\
\hline 13 & & 445.96 & 724.6 & 2.52 & 28.03 & 4.01 \\
\hline 14 & & 606.42 & 1042.6 & 5.39 & 43.68 & 4.26 \\
\hline 15 & & 269.99 & 392.16 & 1.10 & 13.79 & 1.38 \\
\hline 16 & & 412.08 & 653.58 & 3.06 & 25.29 & 1.19 \\
\hline
\end{tabular}

Индекс связанности второго порядка рассчитывали по формуле

$$
{ }^{2} \chi=\sum_{q=1}^{L}\left(\delta_{\mathrm{i}} \delta_{\mathrm{j}} \delta_{\mathrm{k}}\right)^{-1 / 2}
$$

где $i, j, k$ - атомы молекулы, образующие две соседние связи; L - число таких двух соседних связей. При этом $\delta_{i}$ определяли как $\delta_{i}=Z_{i}-h_{i}$, где $Z_{i}-$ число электронов валентной оболочки; $\mathrm{h}_{\mathrm{i}}$ - число атомов водорода, связанных с атомом $\mathrm{i}$.

Индексы связанности более высоких порядков рассчитывали по аналогичным известным формулам [14]. Расчет индекса Винера (ИВ) проводили с помощью программы Marvin Sketch Version 15.11.23. Полученные значения топологических индексов (ТИ) приведены в таблице 2.

Таблица 2. Значения ТИ исследованных соединений

\begin{tabular}{|c|c|c|c|c|c|c|c|}
\hline № & ${ }^{0} \chi$ & ${ }^{1} \chi$ & ${ }^{2} \chi$ & ${ }^{3} \chi$ & ${ }^{4} \chi$ & ${ }^{5} \chi$ & $\mathrm{W}$ \\
\hline 1 & 8.408 & 4.600 & 2.541 & 1.267 & 0.660 & 0.324 & 454 \\
\hline 2 & 10.587 & 6.054 & 3.416 & 1.823 & 0.844 & 0.451 & 826 \\
\hline 3 & 13.896 & 8.404 & 4.527 & 2.334 & 1.192 & 0.630 & 1585 \\
\hline 4 & 5.623 & 3.087 & 1.668 & 0.921 & 0.443 & 0.189 & 153 \\
\hline 5 & 6.545 & 3.259 & 1.829 & 0.908 & 0.394 & 0.194 & 191 \\
\hline 6 & 8.391 & 4.107 & 2.296 & 1.242 & 0.536 & 0.264 & 294 \\
\hline 7 & 12.242 & 6.414 & 3.510 & 1.857 & 0.995 & 0.504 & 1143 \\
\hline 8 & 5.493 & 2.953 & 1.334 & 0.637 & 0.311 & 0.157 & 153 \\
\hline 9 & 12.111 & 6.872 & 3.530 & 1.858 & 0.937 & 0.496 & 1143 \\
\hline 10 & 9.623 & 5.101 & 2.788 & 1.468 & 0.769 & 0.335 & 499 \\
\hline 11 & 14.396 & 8.680 & 4.584 & 2.479 & 1.233 & 0.622 & 1771 \\
\hline 12 & 8.700 & 4.719 & 2.514 & 1.277 & 0.679 & 0.338 & 405 \\
\hline 13 & 10.545 & 5.816 & 3.201 & 1.702 & 0.793 & 0.375 & 568 \\
\hline 14 & 15.319 & 9.111 & 4.818 & 2.655 & 1.316 & 0.654 & 1923 \\
\hline 15 & 4.742 & 2.749 & 1.493 & 1.084 & 0.440 & 0.216 & 79 \\
\hline 16 & 8.566 & 4.966 & 2.812 & 1.563 & 0.871 & 0.407 & 435 \\
\hline
\end{tabular}




\section{Обсуждение результатов}

В соответствии с основными закономерностями обращенно-фазового режима жидкостной хроматографии удерживание, как правило, увеличивается с ростом поляризуемости, объема и площади поверхности молекул сорбатов в результате усиления дисперсионных взаимодействий с неполярным сорбентом. Известные к настоящему времени хроматографические модели, к сожалению, не вполне учитывают природу обращенно-фазового сорбента, определяя его исключительно как «неполярный» [15]. Однако развитие ВЭЖХ в современных условиях привело к созданию новых поколений сорбентов, неполярных с точки зрения хроматографической полярности, но обладающих некоторыми специфическими свойствами за счет особенностей их структуры или методов синтеза $[16,17]$. Примерами таких сорбентов являются сверхсшитый полистирол (ССПС) и пористый графитированный углерод (ПГУ), свойства которых достаточно хорошо описаны в литературе. Одной из характеристик таких сорбентов является их плоская поверхность, способствующая более тесному контакту с плоскими же молекулами сорбатов и, таким образом, лучшему удерживанию последних на поверхности сорбента по сравнению с неплоскими молекулами. Очевидно, что при сорбции на поверхности таких сорбентов наряду с физико-химическими параметрами, определяющими межмолекулярные взаимодействия в хроматографической системе, важной становится роль топологии молекул аналитов.

Известно, что топологические индексы достаточно широко используются в оценке физико-химических свойств соединений, для которых существенное значение имеют размеры молекул и степень их разветвленности, то есть параметры, в значительной степени определяющих энергию межмолекулярных взаимодействий [14]. При этом базисом для определения как физико-химических характеристик соединений, так и их строения является наличие соответствующих корреляций между структурой и свойством. Однако большое разнообразие используемых ТИ приводит к тому, что для конкретного применения тип подходящих ТИ, как правило, выбирают методом проб и ошибок. Поэтому сравнение и оценка их дискриминирующей и прогнозирующей способности по отношению к различным классам соединений является весьма важной проблемой, решение которой позволит унифицировать систему ТИ при описании структуры и свойств молекул, а также корреляций между ними.

Объектами нашего исследования явились соединения, в молекулах которых присутствуют два азотсодержащих фрагмента с различным сочетанием имидазольного или бензимидазольного, триазольного или триазинового остатков. Таким образом, основной особенностью строения этих аналитов является наличие в их структуpe 5-6 атомов азота, которые оказывают существенное взаимное влияние внутри единой ароматической и в общей молекулярной системах (для сравнения нами были также взяты производные 1,3-бензоксазола). Результатом такого влияния оказывается высокая степень делокализации $\pi$-электронов, приводящая к электроноизбыточности производных триазола и электронодефицитности производных триазина [5]. Кроме того, даже в отсутствие функциональных групп в структуре исследованных аналитов наличие нескольких гетероатомов и несимметричное строение молекул существенно увеличивает их полярность, о чем свидетельствуют значения дипольного момента, приведенные в таблице 1 . В сравнительно широких пределах изменяются и остальные характеристики молекул, включая топологические индексы (таблица 2). Значения индексов связанности ${ }^{\mathrm{n}} \chi$ исследованных соединений, как следует из приведенных данных, уменьшаются с ростом их порядка, что соответствует известным представлениям об изменении ИС при уменьшении взаимного влияния атомов в молекуле внутри её топологического пространства, связанном с геометри-

Карасева и др. / Сорбционные и хроматографические процессы. 2018. Т. 18. № 6 
ческим и топологическим удалением атомов в молекуле друг от друга [18]. В целом так же, как и для исследованных нами ранее производных бензотриазола [3], ИС изменяются в соответствии с изменением их физико-химических параметров, что иллюстрирует рис.1. Строгость корреляций при этом оказывается различной и определяется как природой коррелируемой физико-химической характеристики, так и порядком ИС.

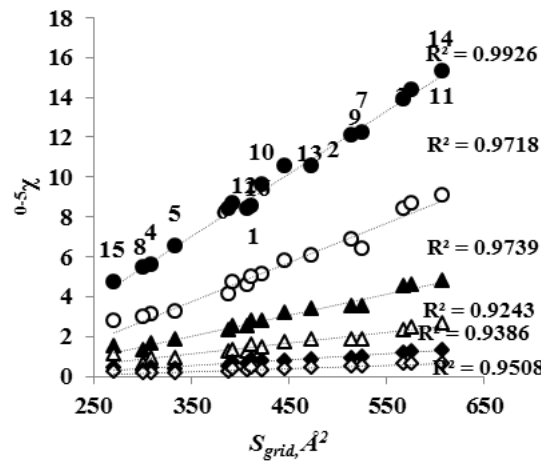

a

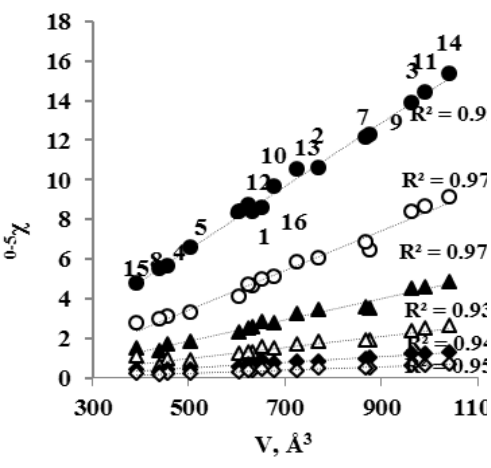

6

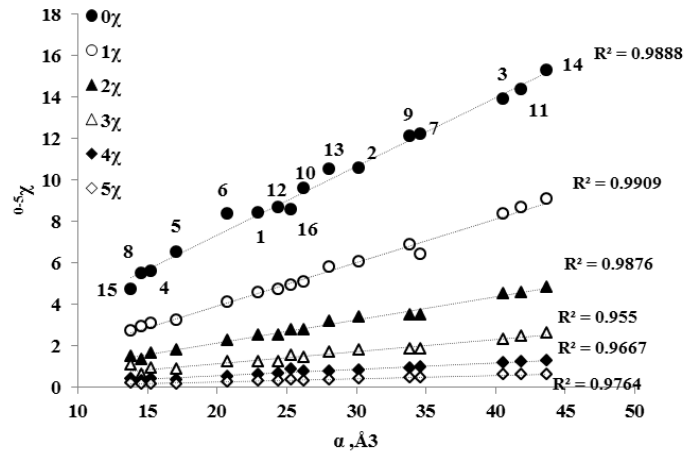

B

Рис. 1. Корреляции между ИС ${ }^{0-5} \chi$, площадью поверхности (а), объемом молекулы (б), поляризуемостью (в).

Анализ полученных корреляций показал, что в целом для исследованных производных 1,2,4-триазола и 1,2,4-триазина с параметрами, характеризующими дисперсионные взаимодействия, хорошо коррелируют ИС всех пяти порядков. В эти же корреляции укладываются и производные 1,3-бензоксазола (вещества 15 и 16). Максимальные значения коэффициента корреляции при этом характерны для корреляций ${ }^{0} \chi$ (значения $R$ в диапазоне 0.995-0.989), минимальные - для ${ }^{3} \chi$ (значения $R$ в диапазоне 0.955-0.924). Следует отметить, что для производных бензотриазола максимальной коррелирующей способностью характеризовались аналогичные корреляции для ИС с нулевого по третий прядок (значения $R$ в пределах 0.995-0.989).

Для исследованных нами соединений практически отсутствует вырождение ИС, и лишь для некоторых близких по структуре веществ (например, 3 и 11, 4 и 8) значения ИС сближаются с ростом n. Для производных бензотриазола нами была установлена обратная зависимость - с ростом порядка связанности дискриминирующая способность ИС по отношению к изомерным производным бензотриазола увеличивалась [3]. Достаточно чувствительными ИС оказываются к размеру гетероцикла и числу атомов азота в них. Так, переход от производных имидазола (вещества 4, 7 ) к производным триазола (вещества 8,9 ) изменяет значения ИС низких порядков примерно на 0.2-0.3 ед. индекса, в то время как индекс Винера для этих соединений оказывается вырожденным. 
Замена имидазольного фрагмента на бензимидазольный (вещества 7 и 11) изменяет ИС нулевого - второго порядков на 1-2 ед.индекса. Индекс Винера при этом также существенно возрастает. В целом значения индекса Винера в ряду исследованных гетероциклов монотонно возрастают с увеличением размеров молекул, однако, корреляция между ИВ и размерными параметрами молекул оказывается несколько менее строгой, чем для ИС (рис. 2).

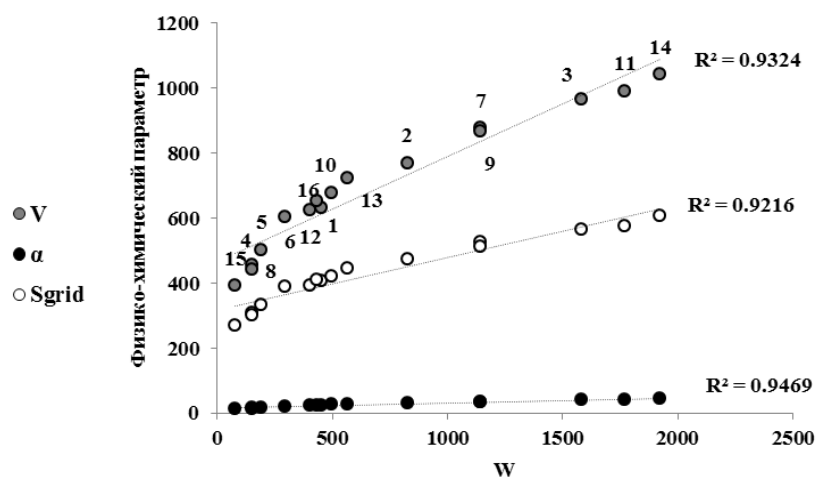

Рис. 2. Корреляция между ИВ и физико-химическими параметрами молекул

Для исследованных производных 1,2,4-триазола и 1,2,4-триазина практически отсутствует вырождение ИС, что благоприятствует их использованию в соответствующих корреляциях. В ряду исследованных нами соединений значения ИС сближаются с ростом порядка индекса (n) лишь для некоторых близких по структуре веществ (например, 3 и 11, 4 и 8), при этом для производных бензотриазола наблюдается обратная зависимость - с ростом порядка связанности дискриминирующая способность ИС по отношению к изомерным производным увеличивается [3]. Достаточно чувствительными ИС оказываются к размеру гетероцикла и числу атомов азота в них. Замена имидазольного фрагмента на бензимидазольный (вещества 7 и 11) изменяет значения ИС малых порядков на 1-2 ед. индекса, а переход от производных имидазола (вещества 4,7 ) к производным триазола (вещества 8,9 ) - примерно на 0.20.3 ед. индекса, в то время как индекс Винера для этих соединений оказывается вырожденным. В целом же значения инкрементов ТИ изменяются в зависимости от порядка ИС и природы фрагмента молекулы, для которого определяется инкремент. Так, $\Delta^{0} \chi$ углеводородных радикалов, как следует из таблицы 3 , остается практически постоянным вне зависимости от положения радикала в молекуле. С ростом порядка ИС значения инкрементов ТИ функциональных групп и радикалов начинают существенно зависеть от положения заместителя, поскольку более высокий порядок ИС определяет характер связывания данного фрагмента (или атома) как с соседними, так и с более удаленными атомами в молекуле [14]. Очевидное сближение инкрементов ИС высоких порядков в связи с уменьшением их значений существенно снижает дискриминирующую способность по отношению к положению заместителя в молекуле. Наибольшую практическую ценность представляют, вероятно, инкременты ИС с нулевого по третий порядок.

Таблица 3. Инкременты индексов связанности

\begin{tabular}{|c|c|c|c|c|c|c|c|}
\hline № вещества & $\Delta^{0} \chi$ & $\Delta^{1} \chi$ & $\Delta^{2} \chi$ & $\Delta^{3} \chi$ & $\Delta^{4} \chi$ & $\Delta^{5} \chi$ & $\Delta \mathrm{W}$ \\
\hline 1 & 2 & 3 & 4 & 5 & 6 & 7 & 8 \\
\hline $3-2$ & 3.309 & 2.350 & 1.111 & 0.511 & 0.348 & 0.179 & 759 \\
\hline $7-4$ & 3.309 & 1.663 & 1.421 & 0.468 & 0.276 & 0.157 & 495 \\
\hline $9-8$ & 3.309 & 1.959 & 1.098 & 0.610 & 0.323 & 0.169 & 495 \\
\hline
\end{tabular}

Карасева и др. / Сорбционные и хроматографические процессы. 2018. Т. 18. № 6 


\begin{tabular}{|c|c|c|c|c|c|c|c|}
\hline 1 & 2 & 3 & 4 & 5 & 6 & 7 & 8 \\
\hline & \multicolumn{5}{|c|}{ Метил-радикал } & & \\
\hline $6-5$ & 0.923 & 0.424 & 0.233 & 0.167 & 0.071 & 0.035 & 51.5 \\
\hline 13-12 & 0.922 & 0.548 & 0.343 & 0.212 & 0.057 & 0.0185 & 81.5 \\
\hline 14-11 & 0.923 & 0.431 & 0.234 & 0.176 & 0.083 & 0.032 & 152 \\
\hline $5-4$ & 0.922 & 0.172 & 0.161 & -0.013 & -0.049 & 0.005 & 38 \\
\hline $13-10$ & 0.922 & 0.715 & 0.413 & 0.234 & 0.024 & 0.040 & 69 \\
\hline & & & Азот & & & & \\
\hline $7-9$ & 0.131 & -0.458 & -0.020 & -0.001 & 0.058 & 0.001 & 0 \\
\hline $4-8$ & 0.130 & 0.134 & 0.334 & 0.284 & 0.132 & 0.032 & 0 \\
\hline
\end{tabular}

Топология молекул, как уже было отмечено ранее, играет важную роль при сорбции из газовой фазы, в то время как ее влияние на сорбцию из водноорганических растворов исследовано в меньшей степени.

В литературе представлены анализ взаимосвязи характеристик удерживания и ТИ с использованием линейного регрессионного анализа и оценка возможности применения уравнения $\lg k=\mathrm{a} \cdot{ }^{\mathrm{n}} \chi+\mathrm{b}$ для аппроксимации этой зависимости [19]. Практически не вызывает сомнений возможность применения данного уравнения для характеристики удерживания сорбатов разного строения на неполярных неподвижных фазах в условиях газовой хроматографии. Для условий жидкостной хроматографии, как следует из представленных в литературе данных, возможность аппроксимирования зависимости удерживания от ТИ с помощью линейного уравнения оказывается в максимальной степени определяемой строением молекул сорбатов, анализируемых в конкретной хроматографической системе [20].

Из полученных нами данных для производных 1,2,4-триазола и 1,2,4-триазина следует, что качество аппроксимации подобных зависимостей линейными уравнениями оказывается различным и определяется, прежде всего, природой сорбента, а также порядком ИС (таблица 3). Максимальным оказывается уровень корреляций для фактора удерживания на ОДС ( $R$ изменяется в диапазоне 0.832-0.905), минимальным - для ПГУ ( $R$ в диапазоне 0.658-0.712). При этом для удерживания на ОДС и ССПС с повышением порядка ИС значения коэффициента $\mathrm{R}$ возрастают, а для удерживания на ПГУ такой закономерности нет, и значения $\mathrm{R}$ изменяются немонотонно (таблица 4). Различия в уровне корреляций для разных сорбентов косвенно свидетельствуют о реализации различных механизмов удерживания, точнее, о различных типах взаимодействий между молекулами сорбатов и поверхностью этих сорбентов. Максимальный уровень корреляции для ОДС обусловлен преобладанием дисперсионных взаимодействий, в реализации которых принимает участие весь объем молекулы сорбата. А ТИ, как известно, лучше коррелируют с параметрами, либо приблизительно аддитивными, либо пропорциональными ван-дер-ваальсову объему молекул [21].

Таблица 4. Значения коэффициента корреляции (R) для зависимости $\lg k=\mathrm{a}^{\mathrm{n}} \chi+\mathrm{b}$

\begin{tabular}{|c|c|c|c|c|c|c|}
\hline & ${ }^{0} \chi$ & ${ }^{1} \chi$ & ${ }^{2} \chi$ & ${ }^{3} \chi$ & ${ }^{4} \chi$ & ${ }^{5} \chi$ \\
\hline $\operatorname{lgk}_{\text {Одс }}$ & 0.8317 & 0.8459 & 0.8726 & 0.9120 & 0.9224 & 0.9050 \\
\hline${ }^{*} \operatorname{lgk}_{\text {ССПC }}$ & 0.6579 & 0.7197 & 0.7843 & 0.8997 & 0.8646 & 0.8446 \\
\hline $\operatorname{lgk}_{П г у}$ & 0.7115 & 0.6860 & 0.7094 & 0.6576 & 0.6964 & 0.6986 \\
\hline $\operatorname{lok}_{\text {ОдС }}$ & 0.8426 & 0.8654 & 0.8944 & 0.9257 & 0.9294 & 0.9193 \\
\hline $\operatorname{lgk}_{\text {ССпC }}$ & 0.7231 & 0.7759 & 0.8299 & 0.9182 & 0.8752 & 0.8709 \\
\hline $\operatorname{lgk}_{\Pi Г у}$ & 0.3992 & 0.3568 & 0.3910 & 0.3247 & 0.3943 & 0.3962 \\
\hline
\end{tabular}

* Состав элюента ацетонитрил - вода 1:1; ${ }^{* *}$ Состав элюента ацетонитрил - вода 3:2

Изменение удерживания некоторых исследованных гетероциклов на разных сорбентах с изменением ИС иллюстрирует диаграмма на рис. 3. При общем возрас-

Карасева и др. / Сорбционные и хроматографические процессы. 2018. Т. 18. № 6 
тании значений фактора удерживания с ростом значений ИС, максимальные величины характерны для удерживания производного 1,2,4-триазина (вещество 12) на ПГУ и фенилсодержащих производных на ССПС (вещества 11 и 14), что, вероятно, обусловлено реализацией на ССПС и ПГУ специфических взаимодействий, дополнительных к дисперсионным - для сверхсшитого полистирола, как известно, это $\pi$ взаимодействия с молекулами сорбатов, имеющими свободные электронные пары, $d$-орбитали или ароматические фрагменты, для пористого графитированного углерода - электростатические взаимодействия полярных молекул адсорбата с индуцированными зарядами на его поверхности. Реализация таких взаимодействий требует определенной ориентации молекул относительно поверхности сорбента и, таким образом, вносит определенные коррективы в характер зависимости удерживания от размерных параметров. Именно в связи с этим обстоятельством значительные размеры молекул (объем и площадь поверхности) и большие величины ТИ не обязательно приведут к максимальному удерживанию на таких сорбентах.

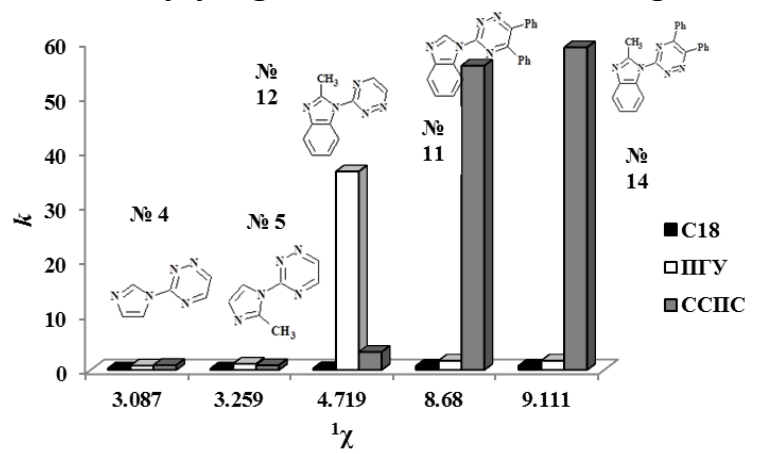

Рис. 3. Соотношение между значениями фактора удерживания исследованных соединений на разных сорбентах и ИС (содержание ацетонитрила в элюенте $80 \%$ об.)

Как следует из приведенных ниже примеров, несмотря на увеличение значений площади поверхности, объема молекулы и ТИ при замене двух метильных радикалов (вещество 13) на два фенильных (вещество 14), удерживание на ПГУ уменьшается.<smiles>Cc1cnc(-c2c(C)cc3ccccc3c2C)cc1C</smiles>

13<smiles>Cc1cc(C)nc(-c2ccccc2)c1</smiles>

14

$$
k_{\text {Пгу }}=90.61 ; \mathrm{S}=445.96 \AA^{2} ; \mathrm{V}=606.42 \AA^{3} \quad k_{\text {пгу }}=1.69 ; \mathrm{S}=724.6 \AA^{2} ; \mathrm{V}=1042.6 \AA^{3}
$$

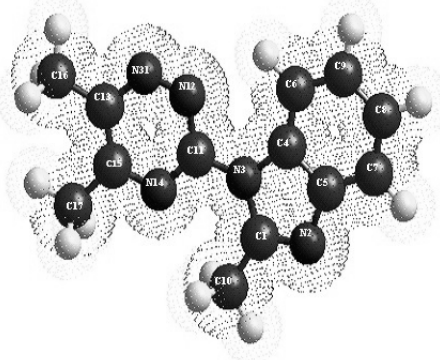

13

Торсионные углы

$<(\mathrm{C} 16, \mathrm{C} 13, \mathrm{~N} 31, \mathrm{~N} 12)=180^{\circ}$

$<(\mathrm{C} 17, \mathrm{C} 15, \mathrm{~N} 14, \mathrm{C} 11)=180^{\circ}$

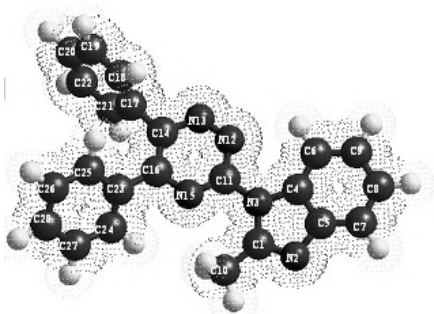

14

$<(\mathrm{C} 21, \mathrm{C} 17, \mathrm{C} 14, \mathrm{C} 16)=-77.29^{\circ}$

$<(\mathrm{C} 14, \mathrm{C} 16, \mathrm{C} 23, \mathrm{C} 24)=135.47^{\circ}$

Рис. 4. Величины торсионных углов соединений 13 и 14 
Вероятной причиной кажущегося несоответствия значений фактора удерживания и размерных характеристик этих веществ может быть стереохимия молекул, не учитываемая как в использованных физико-химических, так и топологических параметрах. И действительно, в соответствии со значениями торсионных углов между углеводородными радикалами и основным гетероциклическим фрагментом молекул (рис. 4), вещество 13 оказывается значительно более плоским по сравнению со своим аналогом 14 и, следовательно, способным к более тесному контакту с плоской поверхностью ПГУ, результатом которого является его большее удерживание на поверхности данного сорбента.

\section{Заключение}

Таким образом, исследование хроматографического удерживания производных 1,2,4-триазина и 1,2,4-триазола из водно-ацетонитрильных растворов на октадецилсиликагеле, пористом графитированном углероде и сверхсшитом политироле позволило установить существенное влияние строения и топологии молекул этих соединений на их сорбционные характеристики. При сорбции на плоской поверхности ПГУ важной оказывается также ориентация сорбируемых молекул относительно сорбента, определяемая их конкурирующими взаимодействиями с сорбентом и элюентом. Результаты моделирования методами молекулярной динамики адсорбционных комплексов, образуемых при сорбции азотистых гетероциклов из объема водноацетонитрильного раствора, мы планируем представить в следующей публикации.

Работа выполнена при поддержке Министерства образования и науки Российской Федерации в рамках государственного задания по гранту № 4.5883.2017/8.9.

\section{Список литературы}

1. Киселев А.В.. Межмолекулярные взаимодействия в адсорбции и хроматографии. М. Высшая школа. 1986. 360 с.

2. Kaliszan R., Straten M.A., Markuszewski M. et al. // J. Chromatorg. A. 1999. Vol. 855. pp. 455-486.

3. Джабиева С.А., Курбатова С.В., Колосова Е.А. // Журн. структ. химии. 2017. Т. 58. № 3. C. 484-493.

4. Некрасова Н.А., Курбатова С.В., Земцова М.Н. // Сорбиионные и хроматографические проиессы. 2016. Т. 16. № 2. С. 154-162.

5. Карасева И.Н., Карасев М.О., Нечаева О.Н., Курбатова С.В. // Журн. физ. химии. 2018. T. 92. № 7. C. 1164-1171.

6. Papandopoulou M.V., Bloomer W.D., Rosenzweig H.S. // Bioorg.\& Med. Chem. 2017. Vol. 25. pp. 6039-6048.

7. Cascioferro S., Parrino B., Spano` V. et al. // Europ. J. of Med. Chem. 2017. Vol. 142. No 13-14. pp. 328-375.

8. Singia P., Luxami V., Paul K. // Europ. J. Medical Chem. 2015. Vol.102. pp. 39-57.

9. Aromí G., Barrios L.A., Roubeau O., Gamez P. // Coord. Chem. Rev. 2011.Vol. 255. pp. 485-546.
10.Browne W.R., Hage R., Vos J. G. // Coord. Chem. Rev. 2006. Vol. 250. No 13-14. pp. 1653-1668.

11.Zhang S., Shi W., Cheng P. // Coord. Chem. Rev. 2017. Vol.352. pp. 108-150.

12. Contreras R., Flores-Parra A., Mijangos E. et al. // Coord. Chem. Rev. 2009. Vol. 253. No 15-16. pp. 1979-1999.

13.Шатц В.Д., Сахартова О.В. Высокоэффективная жидкостная хроматография. Рига. Зинатне. 1988. 390 с.

14.Руврэ Д. В кн. Химические приложения топологии и теории графов. Под ред. Р. Кинга. М. Мир. 1987. С. 183.

15.Polyakova Y.L., Row K.H. // Chromatographia. 2007. Vol. 65. pp. 59-63.

16. Tsyurupa M.P., Davankov V.A. // React. \& Funct. Polym. 2006. Vol. 66. No 7. pp. 768-779.

17. West C., Elfakir C., Lafosse M. // J. Chromatogr. A. 2010. Vol. 1217. No 19. pp. 32013216.

18.Красных Е.Л., Портнова С.В. // Журн. структур. химии. 2016. Т. 57. № 3. С. 466474. 
19.Dashtbozorgi Z. Golmohammadi H., Konoz E. // Chromatographia. 2012. Vol. 75. No 13-14. pp. 701-710.

\section{References}

1. Kiselev A.V. Mezhmolekulyarnye vzaimodejstviya $\mathrm{v}$ adsorbcii i hromatografii, M., Vysshaya shkola Publ., 1986, 360 p.

2. Kaliszan R., Straten M.A., Markuszewski M., Cramers C.A. et al., J. Chromatorg. A., 1999, Vol. 855, No 2, pp.455-486. DOI: 10.1016/S0021-9673(99)00742-6

3. Dzhabieva S.A., Kurbatova S.V., Kolosova E.A. , J. Strukt. Khimii, 2017, Vol. 58, No 3, pp. 484-493.

4. Nekrasova N.A., Kurbatova S.V., Zemtsova M.N., Sorbtsionnye i khromatograficheskie protsessy, 2016, Vol.16, No 2, pp.154-162.

5. Karaseva I.N., Karasev M.O., Nechaeva O.N., Kurbatova S.V., Rus. J. of Physical Chemistry, 2018,Vol. 92, No 7, pp. 1164-1171.

6. Papandopoulou M.V., Bloomer W.D., Rosenzweig H.S., Bioorg. \& Med. Chem., 2017, Vol. 25, pp. 6039-6048. DOI: 10.1016/j.bmc.2017.09.037

7. Cascioferro S., Parrino B., Spano' V. et al., Europ. J. of Med. Chem., 2017, Vol.142, pp. 328-375. DOI: 10.1016/j.ejmech.2017.08.009.

8. Singia P., Luxami V., Paul K., Europ. $J$. Medical Chem., 2015, Vol. 102, pp. 39-57. DOI: 10.1016/j.ejmech.2015.07.037

9. Aromí G., Barrios L. A., Roubeau O., Gamez P., Coord. Chem. Rev., 2011, Vol. 255, pp.485-546. DOI: 10.1016/j.ccr.2010.10.038

10. Wesley R. Browne, R. Hage, Johannes G. Vos ,Coord. Chem. Rev., 2006, Vol. 250, No 13-14, pp. 1653-1668. DOI: 10.1016/j.ccr.2005.12.008.

Карасева Ирина Николаевна - аспирант кафедры физической химии и хроматографии Самарского национального исследовательского университет им. академика С.П. Королева, Самара

Карасев Максим Олегович - к.х.н., старший преподаватель кафедры неорганической химии Самарского национального исследовательского университет им. академика С.П. Королева, Самара, 8-(846) 334-54-45

Курбатова Светлана Викторовна - д.х.н., професcop, декан химического факультета Самарского национального исследовательского университет им. Академика С.П. Королева, Самара
20. Kaliszan R. Quantitative structure chromatographic retention relationships. N.-Y.: John Wiley \& Sons. Inc. 1987. 304 p.

21.Tueros M., Castro E.A., Toropov A.A. // J. Mol. Model. 2001. Vol. 7. pp.178-183.

11. Zhang S., Shi W., Cheng P., Coord. Chem. Rev., 2017, Vol. 352, pp. 108-150. DOI: 10.1016/j.ccr.2017.08.022.

12. Contreras R., Flores-Parra A., Mijangos E. et al., Coord. Chem. Rev, 2009, Vol. 253, No 15-16, pp. 1979-1999. DOI: 10.1016/j.ccr.2009.02.020

13. Shatts V.D., Sakhartova O.V. Vysokoeffektivnaya zhidkostnaya khromatografiya, Riga, Zinatne,1988, 390 p.

14. Ruvre D. V kn. Khimicheskie prilozheniya topologii i teorii grafov / Pod red. R. Kinga, M., Mir, 1987, 183 p.

15. Polyakova Y.L., Row K.H., Chromatographia, 2007, Vol. 65, pp. 59-63. DOI: 10.1365/s10337-006-0119-0.

16. Tsyurupa M.P., Davankov V.A., React. \& Funct. Polym., 2006, Vol. 66, No 7, pp. 768779 .

DOI:

10.1016/j.reactfunctpolym.2005.11.004

17. West C., Elfakir C., Lafosse M., J. Chromatogr. A., 2010, Vol.1217, No 19, pp. 3201-3216.

DOI:

10.1016/j.chroma.2009.09.052

18. Krasnykh E.L., Portnova S.V., Zhurn. struktur. Khimii, 2016, Vol. 57, No 3, pp.466-

474. DOI: $10.15372 / \mathrm{JSC} 20160303$

19. Dashtbozorgi Z. Golmohammadi H., Konoz E., Chromatographia, 2012, Vol. 75, No 13-14, pp. 701-710.

20. Kaliszan R. Quantitative structure chromatographic retention relationships. N.-Y.: John Wiley \& Sons. Inc., 1987, 304 p.

21. Tueros M., Castro E.A., Toropov A.A., J. Mol. Model., 2001, Vol. 7, pp.178-183.

Karaseva Irina N. - post-graduate student at the department of physical chemistry and chromatography, Samara National Research University named after academician S.P. Korolev, Samara

Karasev Maksim O. - Cand. Chem. Sci., senior lecture at the department of inorganic chemistry, Samara National Research University named after academician S.P. Korolev, Samara

Kurbatova Svetlana V. - dean of the chemical faculty, professor, Samara National Research University named after academician S.P. Korolev, Samara, E-mail: curbatsv@gmail.com 